\title{
Self-Expandable Transcatheter Aortic Valve Frame Infolding: An Increasingly Recognized Complication
}

\author{
Gurpreet Singh ${ }^{1}$, Vien Le ${ }^{1}$, Robert J Wiechmann², Steven W Schreiter ${ }^{1}$ \\ ${ }^{1}$ Department of Cardiovascular Medicine, Mayo Clinic Health System, Eau Claire, WI, USA \\ ${ }^{2}$ Department of Cardiovascular Surgery, Mayo Clinic Health System, Eau Claire, WI, USA
}

Doi: 10.12890/2020_002100 - European Journal of Case Reports in Internal Medicine - ๑ EFIM 2020

Received: 25/10/2020

Accepted: $14 / 11 / 2020$

Published: $14 / 12 / 2020$

How to cite this article: Singh G, Le V, Wiechmann RJ, Schreiter SW. Self-expandable transcatheter aortic valve frame infolding: an increasingly recognized complication. EJCRIM 2020;7: doi:10.12890/2020_002100.

Conflicts of Interests: The Authors declare that there are no competing interests.

This article is licensed under a Commons Attribution Non-Commercial 4.0 License

\section{ABSTRACT}

Transcatheter aortic valve replacement (TAVR) with either a balloon-expandable or a self-expandable transcatheter heart valve (THV) is an approved therapy for patients with symptomatic severe aortic stenosis and high or intermediate surgical risk. Here we present a case of severe valve frame infolding of a CoreValve Evolut $\mathrm{PRO}^{\circledR}$ self-expandable THV (Medtronic Inc.), which was restored to optimal geometry with balloon post-dilation.

\section{LEARNING POINTS}

- Clinicians should be aware of the rare complication of frame infolding during deployment of a self-expanding transcatheter valve.

- Multimodality cardiac imaging is important to optimize transcatheter valve deployment.

\section{KEYWORDS}

Transcatheter aortic valve replacement, cardiac CT scan

\section{CASE DESCRIPTION}

A 93-year-old man with symptomatic severe aortic stenosis (AS) was referred to our centre for transcatheter aortic valve replacement (TAVR). Pre-procedural computed tomography (Fig. 1) showed severe trileaflet aortic valve calcification, and extensive calcium extending from the aortic annulus to the left ventricular outflow tract (LVOT). Our heart team decided to proceed with a CoreValve Evolut PRO ${ }^{\circledR}$ selfexpandable TAVR (Medtronic Inc. Minneapolis, MN, USA).

The procedure was done under general anaesthesia, and left femoral access was used to advance the equipment. Balloon valvuloplasty was performed with a $20 \mathrm{~mm}$ Z-MED balloon (Braun Medical), and an Evolute PRO ${ }^{\circledR}$ valve was then advanced under fluoroscopic guidance. During the procedure, for better positioning we had to recapture the valve twice. After the first recapture, the valve struts looked malaligned. We expected this abnormality to get better after complete deployment, but that did not happen (Fig. 2, left image). A post-procedural echocardiogram and non-contrast ECG-gated CT confirmed severe periprosthetic regurgitation, a high prosthetic gradient (systolic mean Doppler gradient $30 \mathrm{mmHg}$ ), and severe infolding of the CoreValve stent frame from the inflow to the waist (Fig. 3, left image; Fig. 4, left image). Our heart team decided to take the patient back to the hybrid operating room for redo balloon valvuloplasty in an attempt to restore the frame geometry and improve transcatheter heart valve (THV) function. We used an Edwards 23 mm balloon catheter, which restored the optimal geometry of the CoreValve frame (Fig. 2, right image; Fig. 4, right image) and significantly improved the periprosthetic regurgitation from severe to mild (Fig. 3, right image) and systolic mean Doppler gradient from 30 to $11 \mathrm{mmHg}$. 

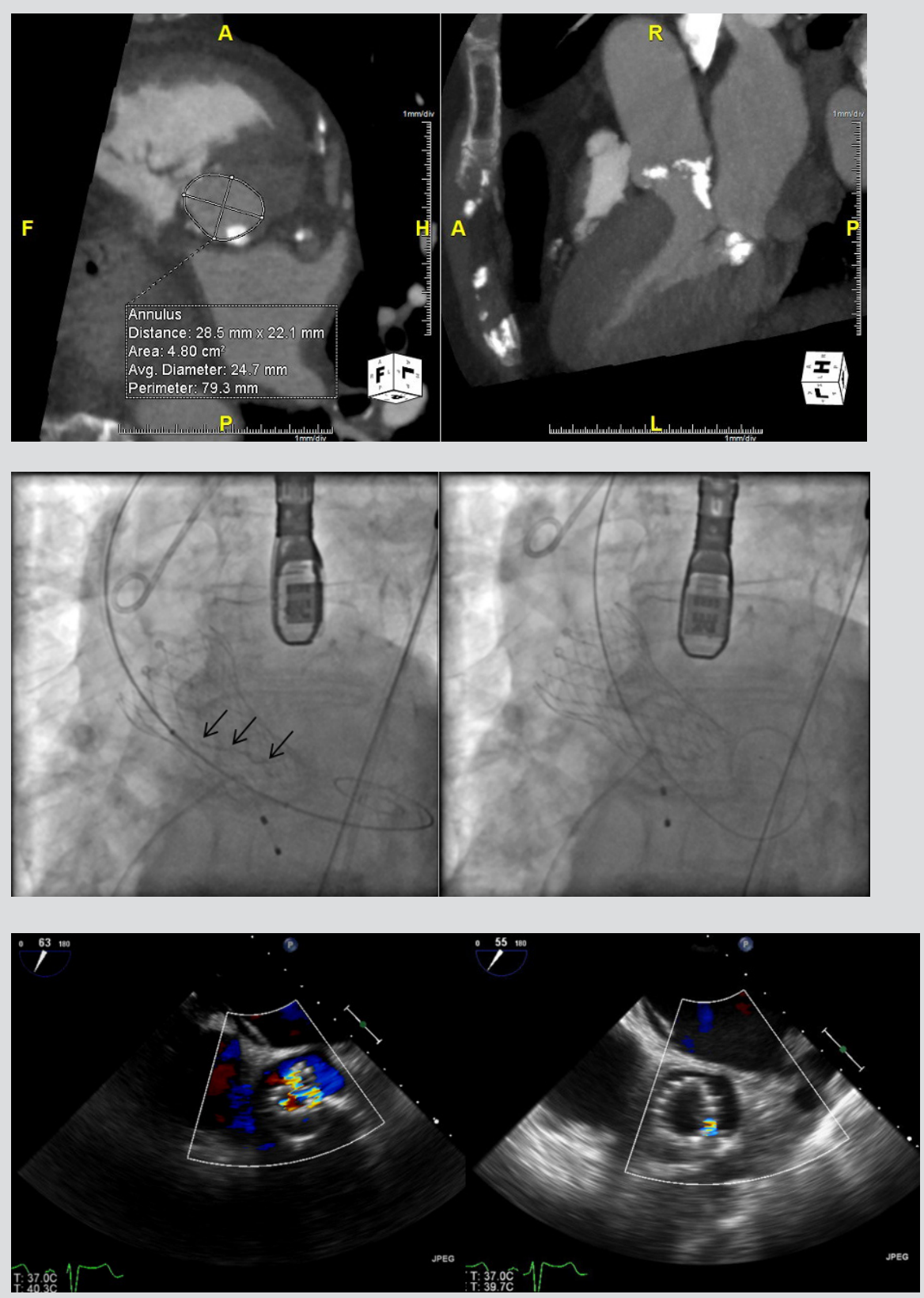

Figure 3. Echo images showing improvement in aortic

regurgitation (before valve ballooning in the left image, and after ballooning in the right image)

Figure 2. Fluoroscopy images. Black arrows indicate infolding of the transcatheter valve (left image), which resolved after ballooning of the valve (right image)

Figure 1. Aortic annulus with eccentric calcification

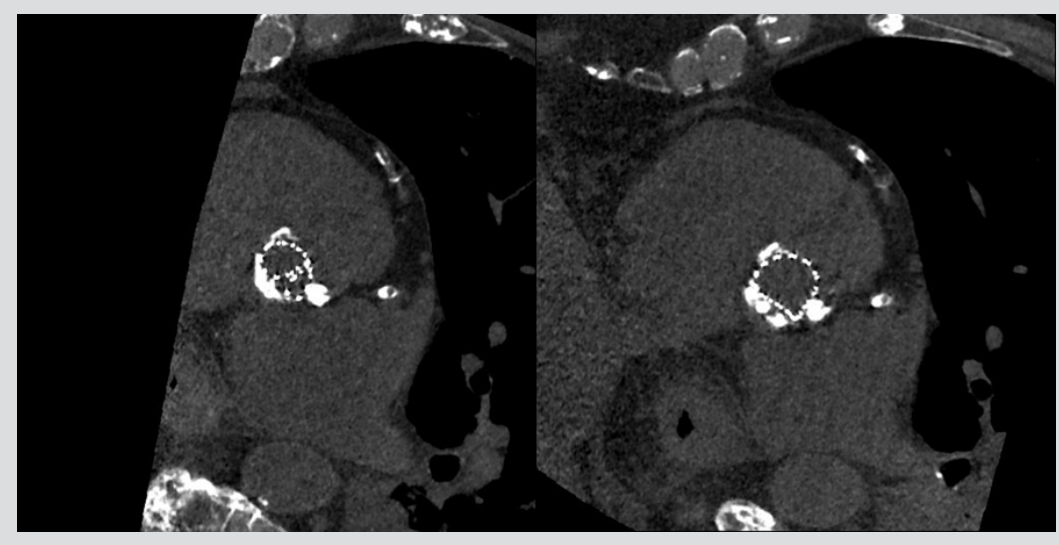




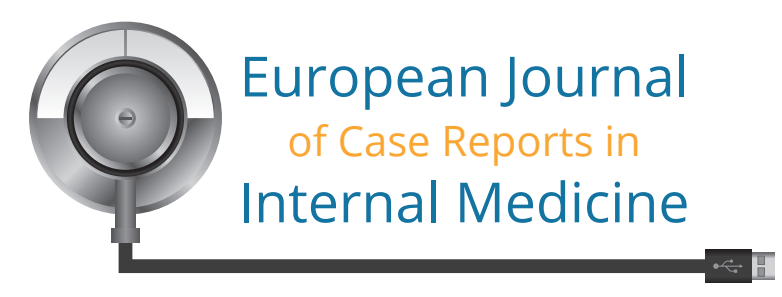

\section{DISCUSSION}

The distortion of the valve frame was the result of eccentric calcification causing asymmetric recapturing, which later on resulted in infolding after subsequent deployment. This issue can arise due to eccentric calcium, valve oversizing, and improper loading of the valve ${ }^{[1,2]}$. It can also occur during recapture when trying to deploy the valve in a better position as demonstrated by this case. Our case highlights that infolding in a self-expanding valve is unlikely to resolve on its own considering that we waited for few days to allow it to self-correct.

We suggest that after a recapture, the CoreValve should be carefully examined to rule out any distortions. The valve should be replaced with a new one in case of asymmetric recapture, because the valve may or may not be able to expand fully after release. However, if the valve has been deployed, as demonstrated by this case, balloon valvuloplasty with an appropriately sized balloon should be considered. The valve should be crossed with a pigtail to avoid paravalvular crossing or through the valve struts. There is a theoretical possibility of deploying the valve inside the valve to increase the outward radial force, but it is difficult to comment on the utility of this approach considering the lack of literature.

\section{REFERENCES}

1. Kaple RK, Salemi A, Wong SC. Balloon valvuloplasty treatment of an infolded CoreValve. Catheter Cardiovasc Interv 2017;89:499-501.

2. Ben-Dor I, Rogers T, Satler LF, Waksman R. A word of caution using self-expanding transcatheter aortic valve-frame infolding. Catheter Cardiovasc Interv 2019;93:555-558. 